donating a methyl group, thus converting homocysteine to methionine. Limited literature exists outlining the risk of metabolic encephalopathy associated with hypermethioninaemia presenting in children with classical Homocystinuria. Here we present one such case, made more remarkable by the absence of treatment with betaine.

Case description Our patient was diagnosed on newborn screening on day 4 of life with classical Homocystinuria and had been promptly commenced on a treatment regime. At four weeks of age he presented to his local hospital with poor feeding, vomiting, and lethargy. Following this acute presentation, he was started on IV antibiotics but a source of infection could not be established. Hyponatraemia, hypertension and proteinuria were noted. His renal ultrasound revealed increased echogenicity bilaterally with no evidence of renal vein thrombosis. His brain magnetic resonance imaging (MRI) showed areas of diffusion restriction bilaterally with normal MR spectroscopy and MR angiogram. Intensive care treatment was required, including intubation and ventilation, and antihypertensive treatment. No underlying thrombo-embolic cause could be established. His blood tests which were taken at the time of his acute presentation to his local hospital revealed a methionine of $1329 \mu \mathrm{mol} / \mathrm{L}$ with a normal cystine $(23 \mu \mathrm{mol} /$ $\mathrm{L})$ and a raised total homocysteine $(118 \mu \mathrm{mol} / \mathrm{L})$ and free homocysteine $(11 \mu \mathrm{mol} / \mathrm{L})$, it was therefore concluded that his presentation was metabolic in origin. These levels improved promptly with further natural protein restriction, increased calorie intake, including intravenous dextrose and electrolytes. The patient made a full recovery; his brain MRI findings normalised.

Discussion Hypermethioninaemic encephalopathy is a rare complication of classical Homocystinuria. It has previously been described in association with betaine treatment. We are unaware of any previous publications of this complication in patients not on this medication. In addition to monitoring for long-term complications, we suggest that methionine should be monitored regularly in patients with classical Homocystinuria, in particular during any episodes of acute deterioration.

\section{P425 SURGICAL MANAGEMENT OF MUCOPOLYSACCHARIDOSIS -RELATED SPINAL DEFORMITIES}

Polina Ochirova*, Sergey Ryabykh, Alexander Gubin. Russian Ilizarov Scientific Center, Kurgan, Russian Federation

\subsection{6/archdischild-2019-epa.761}

Study design Retrospective cohort study.

Class IV of evidence.

Introduction Spinal deformity in patients with mucopolysaccharidosis a problem requiring early treatment

Material and methods We analyzed 7cases of mucopolysaccharidosis (MPS), treated in Ilizarov Center in 2012 - 2018. There were 1 patient with MPS IH-type, 3 patients with IVAtype and 3 patients with MPS type VI. The age of the patients ranged from 6 to 26 years-old (average age - 10.3 years-old). All patients had spinal deformity with primary and/ or secondary stenosis of the spinal canal. Various surgical treatments were used: 1) two staged surgery was perfomed in one patient with cervical myelopathy and scoliosis: $\mathrm{C} 0-\mathrm{C} 7$ decompression with occipitospondylodesis (OSD) firstly and dual growing rod construction secondly; 2) final fusion in 2 patients with scoliosis; 3) decompression at the stenosis level (cervical spine) with OSD in 4 patients with cervical myelopathy.

Results Scoliosis value was from $20^{\circ}$ to $65^{\circ}$ Cobb, kyphosis from $15^{\circ}$ to $80^{\circ}$ Cobb. Four patients had vertebral stenosis and myelopathy (Frankel C). All patients had pulmonary and cardiac dysfunctions: vital capacity of the lungs was from $21 \%$ to $50 \%$ and abnormal ECG. Imbalance in the frontal and sagittal planes was present in $60 \%$ of patients. Through surgical treatment correction of the kyphosis was by $68 \%$ and scoliosis by $85 \%$. In $4(50 \%)$ patients with spinal stenosis neurologic status improved to Frankel E.

Conclusion Patients with MPS quite often have vertebral stenosis and myelopathy, in such patients with combined stenosis of the spinal canal, it is necessary to combine decompression and fusion.

\section{P426 PROFOUND METABOLIC ACIDOSIS AND HYPERTRIGLYCERIDAEMIA IN MITOCHONDRIAL 3- HYDROXY-3-METHYLGLUTARYL-COA (HMG-COA) SYNTHASE- 2 DEFICIENCY (HMGCS2D)}

${ }^{1}$ Tracey A Conlon*, ${ }^{2}$ Patricia E Fitzsimons, ${ }^{1}$ Abhidhamma Kaninde, ${ }^{2}$ Ingrid Borovickova, ${ }^{1}$ Ellen Crushell. 'National Centre for Inherited Metabolic Disorders, Children's University Hospital, Temple Street, Dublin, Ireland; 'Department of Paediatric Laboratory Medicine, Children's University Hospital, Temple Street, Dublin, Ireland

\subsection{6/archdischild-2019-epa.762}

Introduction Mitochondrial HMG-CoA synthase deficiency (HMGCS2D) is an autosomal recessive disorder of ketone body synthesis characterised by hypoketotic hypoglycaemia, vomiting and hepatomegaly typically in the first year of life.

Methods We report a previously well, 21-month old, unvaccinated male, of non-consanguineous Polish heritage, who presented with encephalopathy, hepatomegaly, severe anion-gap metabolic acidosis and mild hyperammonaemia following a brief intercurrent illness. While there was no documented hypoglycaemia, the sample was markedly lipaemic with Triglycerides 34 $\mathrm{mmol} / \mathrm{L}$. Unlike a similar, recently published case, he made a full neurological recovery.

Results Urine organic acid analysis revealed marked ketonuria, very marked dicarboxylic aciduria and notable increase in medium chain 3-oxodicarboxylic acids. Glutarate was markedly elevated with mild increase in 3-hydroxyglutarate (3HG). 4-hydroxy-6-methyl-2-pyrone and four other putative biomarkers for HMGCSD2 were detected. DBS acetylcarnitine was raised on presentation. Acidosis and hypertriglyceridaemia gradually resolved with intravenous dextrose and bicarbonate. 3HG was slow to normalize while pyrone remained detectable. Molecular genetic analysis of the HMGCS2 gene identified compound heterozygosity for c. $634 \mathrm{G}>\mathrm{A}$ and c. $1016+1 \mathrm{G}>\mathrm{A}$, not previously reported with this phenotype.

Conclusions HMGCS2D should be considered in a child with severe unexplained metabolic acidosis even without hypoglycaemia. The condition should be added to the differential diagnosis of raised urinary glutarate and $3 \mathrm{HG}$. 